\title{
Application of a new technique, spiral tissue microarrays constructed using needle biopsy specimens, to prostate cancer research
}

\author{
AKIRA KOMIYA $^{1}$, TOMONORI KATO ${ }^{1}$, TAKASHI HORI ${ }^{2}$, \\ JUNYA FUKUOKA $^{3}$, KENJI YASUDA ${ }^{1}$ and HIDEKI FUSE ${ }^{1}$ \\ ${ }^{1}$ Department of Urology, Graduate School of Medicine and Pharmaceutical Sciences for Research, \\ University of Toyama; ${ }^{2}$ Laboratory of Pathology, Toyama University Hospital, Toyama; \\ ${ }^{3}$ Department of Pathology, Nagasaki University Graduate School of Biomedical Sciences, Nagasaki, Japan
}

Received August 29, 2013; Accepted October 19, 2013

DOI: 10.3892/ijo.2013.2173

\begin{abstract}
Tissue microarrays were constructed using prostate needle biopsy specimens obtained from 58 patients who underwent radical prostatectomy for localized or locally advanced prostate cancer (PC). We used the spiral array (SA) technique, a novel approach for tissue array construction in a spiral form, which has advantages over small needle biopsy specimens. Roll-shaped tissue pieces produced by slicing a prostate biopsy tissue block and trimming the cancer segment were used to obtain a tissue array block. Cancer segments measuring $>3 \mathrm{~mm}$ were incorporated into the tissue arrays. Cancer fragments $(n=253)$ were obtained from formalinfixed, paraffin-embedded needle biopsy specimens. The median number of cancer fragments per patient was four (1-8, min-max). On SA, the median number of confirmed cancer fragments per patient was four (1-7) and 224 cancer fragments $(88.5 \%)$ were confirmed histologically. Each core of reeled tissue contained at least one cancer fragment. The expressions of multiple prognostic molecular markers for PC (Ki-67, p53 and bcl-2) were immunohistochemically measured using the SA. The Ki-67 and bcl-2 expressions were significantly associated with the Gleason score (GS). A univariate analysis identified Ki-67, bcl-2 and GS as significant predictors of cancer-specific survival, p53 and bcl-2 as significant predictors of overall survival and Ki-67, adjuvant androgen deprivation and GS as significant predictors of biochemical progression. In a multivariate analysis, p53 was independently associated with overall survival, while adjuvant androgen deprivation and GS were associated with biochemical progression. These
\end{abstract}

Correspondence to: Dr Akira Komiya, Department of Urology, Graduate School of Medicine and Pharmaceutical Sciences for Research, University of Toyama, 2630 Sugitani, Toyama 930-0194, Japan

E-mail: komiya@med.u-toyama.ac.jp

Key words: immunohistochemical staining, molecular marker, prostate cancer, spiral array, tissue array results indicate that SA has potential as a new tool for translational research on $\mathrm{PC}$.

\section{Introduction}

Constructing tissue arrays (TAs) from thin needle biopsy specimens is challenging and has not been previously performed in prostate cancer. TAs are a well-established technique, connecting basic research to clinical applications, that have become important as a translational research tool $(1,2)$. This tool has proven to be effective in providing a high-throughput analysis of hundreds of tissue specimens in a single slide, while maintaining uniform experimental conditions and being cost-effective. Tissue samples obtained from multiple patients are processed in an identical manner at the same time. Tissue arrays enable the identification and validation of the functional and clinical significance of physiologic and pathobiologic events regarding gene expression dysregulation, genomic aberrations and epigenetic phenomena by exploring the correlations between molecular information and clinicopathologic features (3-7). This technique has also been applied to prostate cancer research (8-10).

When conventional tissue array (CTA) blocks are constructed, punching tissue cores in 'donor' blocks is required before transferring the blocks into 'recipient' blocks. The donor blocks require adequate tissue to be punched out, and many surgical pathology specimens, especially those obtained from needle biopsies with limited material, are not suitable for CTA construction. This results in exclusion from investigational studies and further selection bias. A needle biopsy specimen may represent the only sample that has been collected from a patient with advanced/metastatic cancer before the administration of medical treatment and/or radiotherapy. We previously reported the findings of conventional immunohistochemical studies of castration-resistant prostate cancer using prostate needle biopsy specimens because tissue specimens could be obtained using diagnostic transrectal ultrasound sonography-guided biopsies only (11-13). In these studies, we were required to stain at least one slide per patient for each molecule examined. 
In this context, we developed a novel approach, termed the spiral array (SA), in which each TA core consists of a reeled layer of tissue cut as a horizontal section from the donor block and not punched as a vertical cylinder core (14). Since the whole section is represented in the SA, sampling bias due to tissue heterogeneity is greatly reduced. SA construction using needle biopsy specimens obtained from breast cancer patients has been described in our previous report (14). Constructing SAs from prostate needle biopsy specimens is challenging because prostate tissue is smaller and thinner than breast cancer tissue. We herein describe in detail the construction and application of a needle biopsy-based TA, a form of SA, for use in prostate cancer research. We also evaluate the significance of known prognostic molecular markers for radical prostatectomy, including Ki-67 $(10,15,16)$, p53 (10,16-18) and bcl-2 (17,19-21), to confirm the potential of the SA as a new tool for translational research on prostate cancer.

\section{Materials and methods}

Patient selection. This study included a total of 58 Japanese patients who were diagnosed as having clinically localized or locally advanced prostate cancer according to the staging procedures performed at our institution, including digital rectal examinations, transrectal ultrasonography, serum prostate specific antigen measurement, pelvic computed tomography, magnetic resonance imaging and/or bone scans. The characteristics of the patients are shown in Table I. The mean age of the patients was $68.0 \pm 4.86$ (mean \pm standard deviation) years. The mean initial serum prostate specific antigen level was $24.0 \pm 25.3 \mathrm{ng} / \mathrm{ml}$ (mean \pm standard deviation). The patients subsequently underwent radical prostatectomy and bilateral pelvic lymphadenectomy targeting the obturator fossa and internal/external iliac region between 1983 and 2008. The median follow-up was 80.9 months (8.0-195.8, min-max). Patients with locally advanced disease underwent neoadjuvant androgen deprivation. Patients with a positive surgical margin in radical prostatectomy specimens received adjuvant external radiotherapy to the prostate bed and/or adjuvant androgen deprivation. Disease progression was defined as an increase in the prostate specific antigen level to $>0.2 \mathrm{ng} / \mathrm{ml}$ or clinical progression.

Construction of the spiral arrays (Fig. 1). A spiral array block holding 58 reeled cores were obtained from prostatic adenocarcinoma cases. We selected formalin-fixed, paraffinembedded (FFPE) blocks holding prostatic adenocarcinoma tissues measuring longer than $3 \mathrm{~mm}$ obtained using prostate needle biopsies in patients undergoing radical prostatectomy from the pathology archive of Toyama University Hospital, Toyama, Japan. The pathological findings, including the Gleason grades in both needle biopsy and radical prostatectomy specimens, were assessed according to the Japanese General Rule for Clinical and Pathological Studies on Prostate Cancer (4th edition, 2010), which includes the 5th edition of the TNM classification (1997). The needle biopsy specimens were obtained using random systematic biopsies performed with an automatic biopsy gun and an 18 gauge $(1.2-\mathrm{mm})$ needle under transrectal ultrasonography guidance. In general, the biopsies comprised 10 peripheral zones. The blocks were
Table I. Background characteristics of the patients.

\begin{tabular}{lr}
\hline Characteristics & $\mathrm{n}$ \\
\hline Age & \\
$\leq 69$ & 36 \\
$\geq 70$ & 22 \\
Initial prostate specific antigen $(\mathrm{ng} / \mathrm{ml})$ & \\
$\leq 10$ & 17 \\
$10.1-20$ & 16 \\
$>20.1$ & 20 \\
Unknown & 5 \\
Clinical T stage & \\
T1c & 12 \\
T2 & 20 \\
T3 & 26 \\
Biopsy Gleason score & \\
6 & \\
7 & 23 \\
8 & 16 \\
$9-10$ & 15
\end{tabular}

Neoadjuvant androgen deprivation

$$
-
$$$$
+
$$

Adjuvant androgen deprivation

$-$

Adjuvant radiotherapy

$-$ 39

$+$

$\mathrm{pN}^{\mathrm{a}}$

$-$

Capsular penetration ${ }^{\mathrm{a}}$

28

$+$

Seminal vesicle invasion ${ }^{\mathrm{a}}$

$\begin{array}{ll}- & 46\end{array}$

$+$

Positive surgical margin ${ }^{\mathrm{a}}$

30

$+$ 28

Ki-67 staining index (\%)

0-9

$\geq 10$

p53

$-, \pm$

$+$

bcl-2

$-, \pm,+$ 50

$++$

${ }^{\mathrm{a}}$ Radical prostatectomy specimens. 


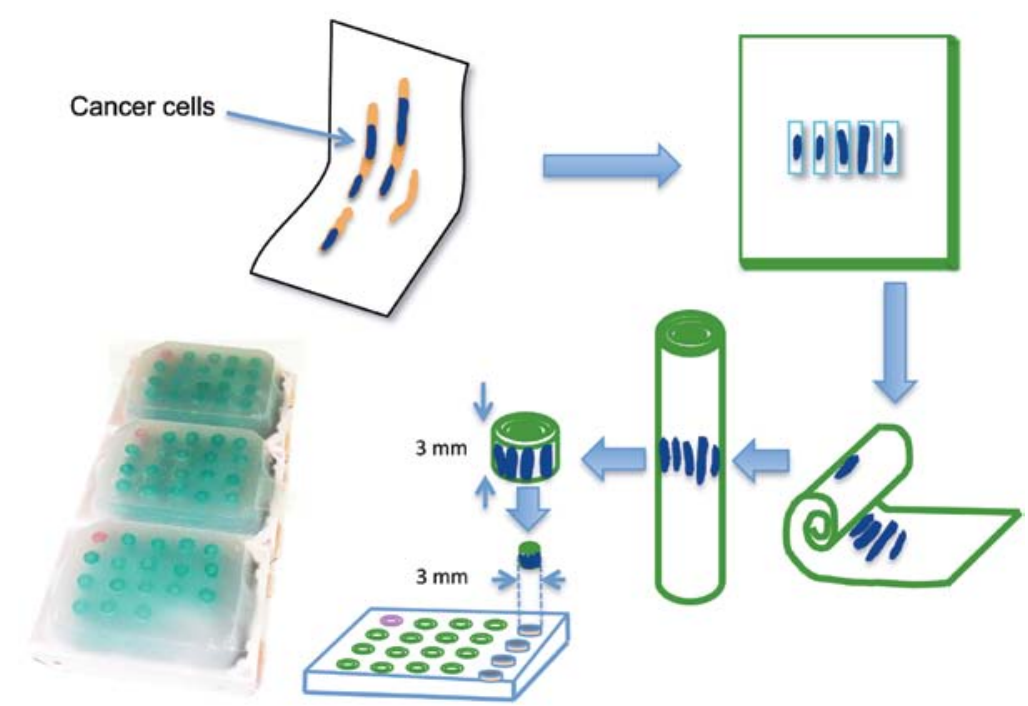

Figure 1. Schematic figure indicating how to make spiral array from prostate core needle biopsy section. Slices, 30- $\mu \mathrm{m}$ thick, of the sample block are cut, cancer cell segments $>3 \mathrm{~mm}$ are trimmed, placed on the plane paraffin sheet and rolled up into cylindrical reels. This cylindrical reel is divided into sections $3 \mathrm{~mm}$ in height and transferred vertically into the recipient block/cassette with arranged 3-mm diameter holes. A spiral array block can then be sectioned in the usual histopathologic fashion.

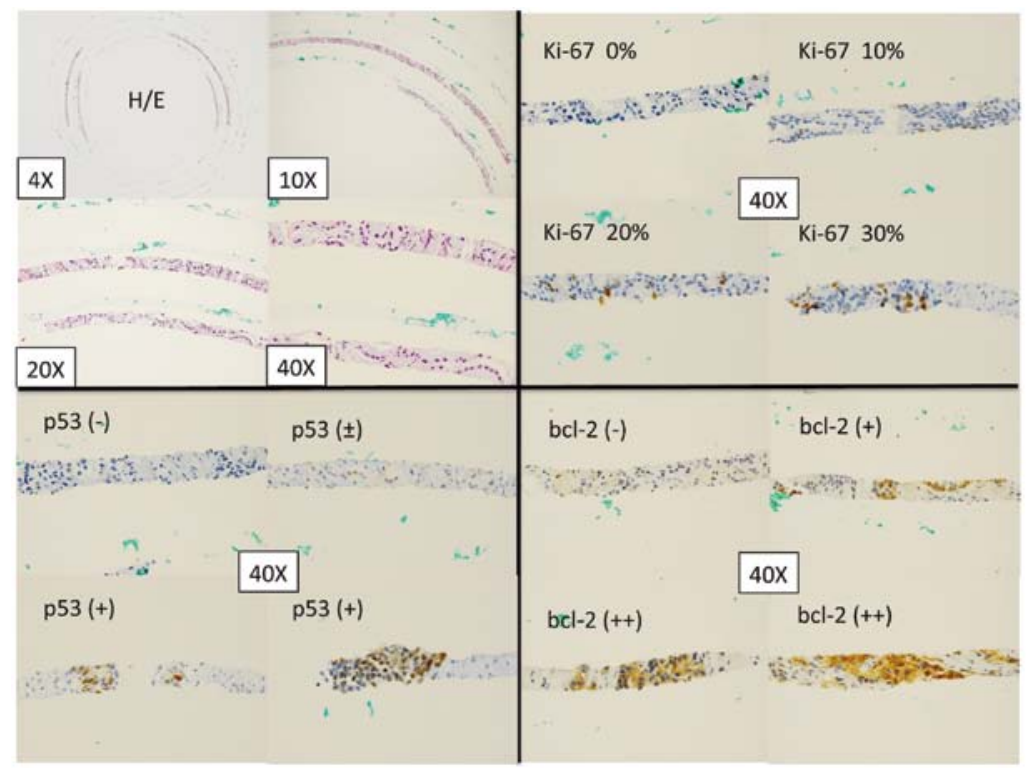

Figure 2. Histologic appearance of the spiral array. H/E, hematoxylin and eosin.

heated to $\sim 40^{\circ} \mathrm{C}$ on the surface, and $30-\mu \mathrm{m}$-thick sections were cut using a standard microtome. A portion of the cancer cell area was trimmed. From the viewpoint of antigen preservation in the paraffin block, the surface layers of the original blocks were discarded. The trimmed tissue sections were heated at $35^{\circ} \mathrm{C}$, flattened and placed on green-dyed intermediated sheets of $20 \mu \mathrm{m}$ in thickness and plane paraffin sheets sectioned at $100 \mu \mathrm{m}$ in thickness from the ordinary plane paraffin blocks (Wako, Osaka, Japan). The melting point was set at $64^{\circ} \mathrm{C}$. Then, the tissue sections, together with the plane paraffin sheet, were rolled up into tissue reels and cut into a 3-mm-tall subcylindrical reel by SA processor (Sakura Finetek Japan, Tokyo, Japan). This process yields $>100$ slices of $4-\mu \mathrm{m}$-thick sections. The subcylinders were cut in the center of the reels without further bias. Each subcylindrical reel was verti- cally inserted into a commercially available tissue-holding cassette made of acrylonitrile/butadiene/styrene polymer with 20 holes, each measuring $3 \mathrm{~mm}$ in diameter (Sakura Finetek Japan). The plastic cassettes were placed on the metal molds (Sakura Finetek Japan) in which the bottom surface of the molds was covered with double-sided adhesive tape. The edges of the inserted subcylindrical reels adhered to the tape. Paraffin melted (Paraffin wax II60, Sakura Finetek Japan) at $65^{\circ} \mathrm{C}$ was poured into the cassette to re-embed the reeled tissue. This step was followed by a cool down period. Lastly, after removing the molds and adhesive tape, the SA block was sectioned at $4 \mu \mathrm{m}$.

Ki-67, p53 and bcl-2 immunohistochemical staining (Fig. 2). Immunohistochemical staining was performed using 
Table II. Associations between the molecular markers and clinicopathological parameters.

\section{P-value}

Clinicopathological factors

vs. Ki-67 vs. p53 vs. bcl-2

$\begin{array}{llll}\text { Age } & 0.549 & 0.579 & 0.105 \\ \text { Initial prostate specific antigen } & 0.055 & 0.206 & 0.369 \\ \text { Clinical T stage } & 0.040^{\mathrm{b}} & 0.247 & 0.283 \\ \text { Biopsy Gleason score } \leq 8 \text { or } \geq 90.0052^{\mathrm{b}} & 0.454 & 0.0115^{\mathrm{b}} \\ \mathrm{pN}^{\mathrm{a}} & 0.146 & 0.476 & 0.078 \\ \text { Capsular penetration }^{\mathrm{a}} & 0.861 & 0.586 & 0.917 \\ \text { Seminal vesicle invasion }^{\mathrm{a}} & 0.113 & 0.236 & 0.210 \\ \text { Positive surgical margin }^{\mathrm{a}} & 0.861 & 0.586 & 0.390 \\ \text { bcl-2 } & 0.0041^{\mathrm{b}} & 0.676 & \\ \text { p53 } & 0.147 & & \end{array}$

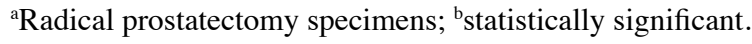

4- $\mu$ m-thick specimens from each block, as previously described (7). Briefly, following sectioning and deparaffinization, the specimens were heated to $95^{\circ} \mathrm{C}$ in a water bath for 40 min in Tris/EDTA buffer at a pH of 9.0 (Target Retrieval Solution, Dako, Kyoto, Japan). A Dako autostainer was used for all immunohistochemical processes. The clones and titra- tions of each antibody were as follows: Ki-67 (clone 30-9, 1:1, Ventana), p53 (clone DO-7, 1:1, Ventana) and bcl-2 (clone bcl2/ A4, 1:50, Bio SB). The EnVision ${ }^{+}$System (Dako) was used for signal enhancement. The reaction products were visualized with $\mathrm{DAB}^{+}$(diaminobenzidine $^{+}$) (Dako). The nuclei were lightly counterstained with Mayer hematoxylin solution. All procedures were carried out at room temperature. Nuclear staining of Ki-67 and p53 was considered positive. The Ki-67 staining index was defined as the percentage of positive nuclei of cells counted using an eyepiece grid. The p53 staining intensity was graded as negative, weakly positive or positive. The Bcl-2 staining intensity was graded as negative, weakly positive, positive or strongly positive, according to the previously reported scoring system $(6,7)$. One investigator (T. Hori) counted the $\mathrm{Ki}-67$-positive nuclei and graded the p53 and bcl-2 immunohistological intensity without prior knowledge of the patient's prognosis-related information. AMACR and p63 staining was also performed to confirm the presence of prostate cancer cells in the SA (data not shown).

Statistical analyses. All statistical analyses were performed using the StatView 5.0 software program (Abacus Concepts, Inc., Berkeley, CA, USA). Student's t-test (continuous variables) or the Mann-Whitney U test (categorical variables) were used to analyze the associations between several clinicopathological factors and the expression levels of the molecular markers. The biochemical progression-free survival rates, cancer-specific survival rates and overall survival rates

\section{A Biochemical progression-free survival}
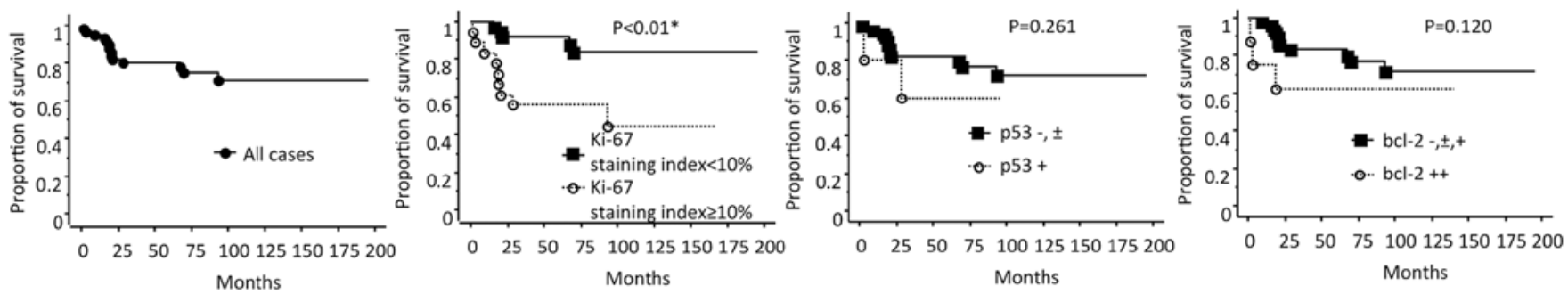

\section{B Cancer-specific survival}
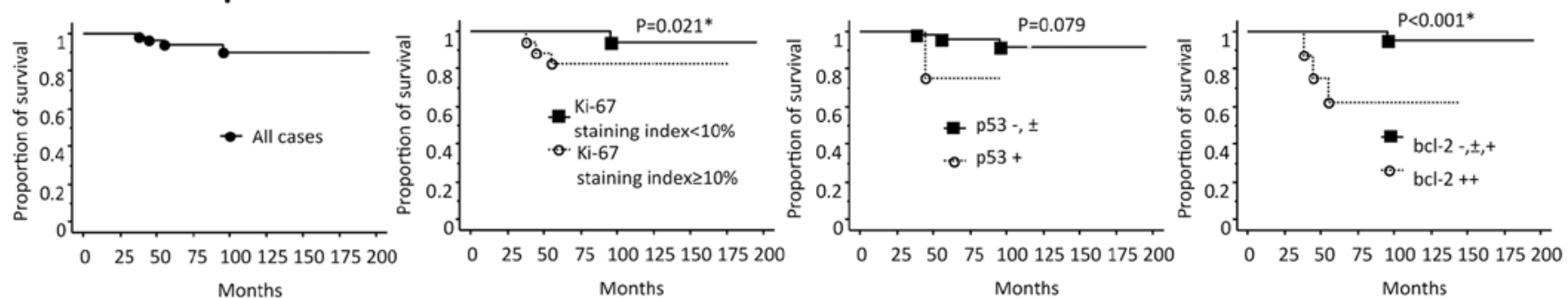

\section{Overall survival}
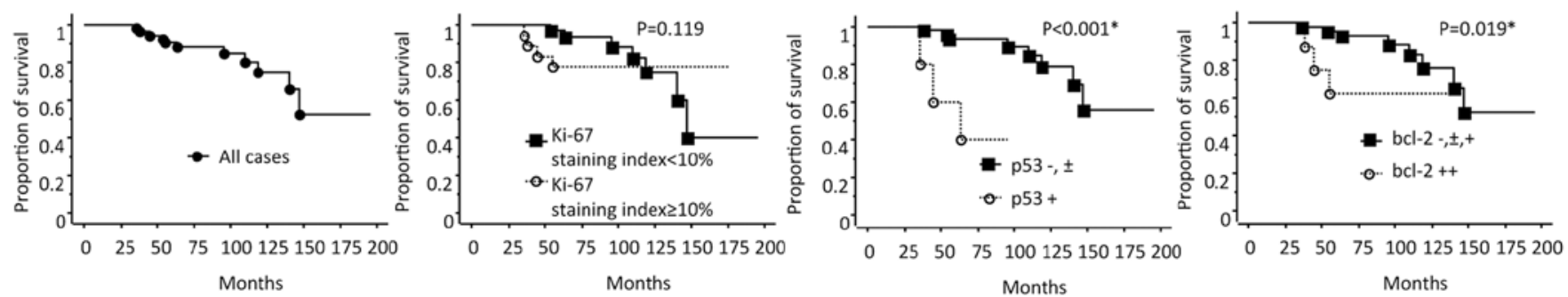

Figure 3. Biochemical progression-free, cancer-specific and overall survival curves according to Ki-67, p53 and bcl-2 staining. 
were calculated according to the Kaplan-Meier method and differences were determined using the Wilcoxon test. The prognostic significance of certain factors was assessed using a Cox proportional hazards regression model. Probability (P) values of $<0.05$ were considered to be significant. The study was approved by the institutional review board of Toyama University Hospital (\#24-13) and conformed to the principles outlined in the Declaration of Helsinki.

\section{Results}

Construction of the spiral array. A total of 253 fragments of cancer cells were isolated from FFPE needle biopsy specimens obtained from 58 patients undergoing radical prostatectomy. The median number of cancer segments per patient was four (1-8, min-max). On SA, the median number of confirmed cancer fragments per patient was four (1-7, min-max) and 224 fragments of the tumor area $(88.5 \%)$ were confirmed histologically: 7 segments in 5 patients, 6 segments in 6 patients, 5 segments in 9 patients, 4 segments in 12 patients, 3 segments in 13 patients, 2 segments in 8 patients and 1 segment in 5 patients. AMACR/p63 staining was also performed for confirmation. Therefore, each core of reeled tissue contained at least one fragment of a cancer area, and one SA block accommodates 20 cores of reeled tissue. Therefore, we constructed three SA blocks from the samples of the 58 patients.

Histopathological parameters and molecular markers in the spiral array and survival. Of the three molecules, Ki-67 and bcl-2 were significantly associated with the Gleason score (GS) (Table II). A univariate analysis identified Ki-67, GS and adjuvant androgen deprivation as significant predictors of biochemical progression, Ki-67, bcl-2 and GS as significant predictors of cancer-specific survival and p53 and bcl-2 as significant predictors of overall survival. Of these significant factors, the p53 expression was found to be independently related to overall survival and GS and adjuvant ADT were related to biochemical progression according to a multivariate analysis (Table III and Fig. 3).

\section{Discussion}

This is the first report on the construction of a TA using prostate needle biopsy specimens. Most of the cancer segments trimmed from the FFPE blocks were histologically confirmed on the SA. Immunohistological staining for molecular markers of prostate cancer revealed prognostically significant findings, consistent with the results of previous reports in the radical prostatectomy setting regarding Ki-67 $(10,15,16)$, p53 (10,16-18) and bcl-2 (17,19-21). These molecular markers are generally used as prognostic factors for biochemical progression following radical prostatectomy. In the present study, a univariate analysis showed the biopsy Gleason score, adjuvant androgen deprivation and $\mathrm{Ki}-67$ staining index to be prognostic factors for biochemical progression. However, the Ki-67 staining index was not demonstrated to be an independent prognostic factor. This may be due to the significant association between the biopsy Gleason score and the Ki-67 staining index, with a higher hazard ratio for the Gleason score
(Table II). As prognostic factors for cancer-specific survival, the biopsy Gleason score, Ki-67 staining index and bcl-2 expression were found to be significant in a univariate analysis. Possibly due to the significant associations between these three factors, none were found to be independent prognostic factors in a multivariate analysis. As prognostic factors for overall survival, the p53 and bcl-2 expressions were found to be significant in a univariate analysis. According to a multivariate analysis, only the p53 expression was an independent factor, with a hazard ratio of 13.3 for overall survival, which has not been previously reported in the literature. Other variables are usually important for survival; however, androgen deprivation and radiotherapy can affect the outcomes $(22,23)$. Based on these results, the immunohistochemical findings obtained with the SA can provide valuable information in addition to conventional clinicopathological parameters. Furthermore, this new technique facilitates further TA construction using prostate needle biopsy specimens in patients who have undergone radiotherapy, androgen deprivation or chemotherapy for localized, locally advanced, metastatic or castration-resistant prostate cancer for whom large surgical specimens are not available.

We previously introduced the SA as a new high-throughput technology that addresses tissue heterogeneity (14). In this original report, we demonstrated the non-inferiority and superiority of this new technique, which allows for improved representation of the donor tissue while keeping the architectural details of the donor block intact, compared to that of the CTA. In addition, the morphologic features were compared between the SA and CTA. We created both SA and CTA analyses for 25 lung adenocarcinomas and 50 multiple tumors of various organs $>1 \mathrm{~cm}^{2}$ and $<2 \mathrm{~cm}^{2}$. Sections measuring $100-\mu \mathrm{m}$ thick were cut using a standard microtome for TA construction. The degree of coverage of tissue heterogeneity was examined by observing the range of the staining intensity differences on immunohistochemistry for cytokeratin 7 and epidermal growth factor receptor (EGFR). The degree of morphologic preservation was tested according to the level of accurate prediction of the histopathologic diagnosis and organ type among three pathologists (board-certified surgical pathologists) who were not involved in the actual construction of the SA. The cases were not disclosed to the panel members before the analysis. The SA demonstrated better representation of the range of staining intensity (heterogeneity) for EGFR $(\mathrm{P}=0.01)$. The level of accuracy for predicting the organ type was significantly higher in the SA than in the CTA $(\mathrm{P}=0.047)$. On the other hand, no significant differences were observed between the SA and CTA in detecting the histologic diagnosis, although the number of accurate answers was higher for the SA. Therefore, the results indicated that the SA is not inferior to the ordinary CTA in preserving morphology.

Since the SA uses only one planar tissue section, blocks with little remaining tissue, which cannot be used for CTA, can be included in further studies. In addition, many core needle biopsy samples, such as breast biopsy specimens or transrectal prostate biopsy specimens, as shown in this study, which cannot be routinely used for CTA construction using conventional approaches, are amenable to the SA technique. Some tissues are too thin to be sampled vertically, as they lack sufficient depth to be used as material for CTA. These 
Table III. Univariate and multivariate analyses of the associations between various parameters and biochemical progressionfree (A), cancer-specific (B) and overall survival (C) in the 58 patients with prostate cancer who underwent radical prostatectomy.

A, Biochemical progression-free survival

\begin{tabular}{|c|c|c|c|c|c|c|c|}
\hline \multirow[b]{2}{*}{ Variables } & & \multicolumn{3}{|c|}{ Univariate analysis $^{\mathrm{b}}$} & \multicolumn{3}{|c|}{ Multivariate analysis ${ }^{\mathrm{c}}$} \\
\hline & & HR & $95 \% \mathrm{CI}$ & P-value & HR & $95 \% \mathrm{CI}$ & P-value \\
\hline Age & $<70$ or $\geq 70$ years & 2.27 & $0.633-8.14$ & 0.345 & & & \\
\hline Initial PSA & $<20$ or $\geq 20 \mathrm{ng} / \mathrm{ml}$ & 1.20 & $0.706-2.039$ & 0.505 & & & \\
\hline Clinical T stage & $\mathrm{T} 1-2$ or $\mathrm{T} 3$ & 1.19 & $0.416-3.394$ & 0.854 & & & \\
\hline Biopsy Gleason score & 8 or higher & 6.03 & $2.07-17.5$ & $0.0001^{\mathrm{d}}$ & 5.87 & $1.688-20.47$ & $0.005^{\mathrm{d}}$ \\
\hline Neoadjuvant ADT & - or + & 0.904 & $0.312-2.618$ & 0.7385 & & & \\
\hline Adjuvant ADT & - or + & 0.162 & $0.036-0.733$ & $0.0095^{\mathrm{d}}$ & 0.139 & $0.029-0.661$ & $0.013^{\mathrm{d}}$ \\
\hline Adjuvant radiotherapy & - or + & 1.06 & $0.354-3.172$ & 0.971 & & & \\
\hline $\mathrm{pN}^{\mathrm{a}}$ & 0 or 1 & 0.916 & $0.119-7.068$ & 0.951 & & & \\
\hline Capsular penetration ${ }^{\mathrm{a}}$ & - or + & 0.42 & $0.141-1.257$ & 0.1032 & & & \\
\hline Seminal vesicle invasion ${ }^{a}$ & - or + & 2.42 & $0.811-7.235$ & 0.080 & & & \\
\hline Positive surgical margin ${ }^{\mathrm{a}}$ & - or + & 0.742 & $0.257-2.140$ & 0.828 & & & \\
\hline $\mathrm{Ki}-67$ staining index & $<10$ or $\geq 10 \%$ & 4.75 & $1.589-14.18$ & $0.0012^{\mathrm{d}}$ & 2.11 & $0.607-7.30$ & 0.240 \\
\hline p53 &,- \pm or + & 2.11 & $0.469-9.492$ & 0.261 & & & \\
\hline bcl-2 &,,- \pm+ or ++ & 1.91 & $0.530-6.879$ & 0.118 & & & \\
\hline
\end{tabular}

B, Cancer-specific survival

\begin{tabular}{|c|c|c|c|c|c|c|c|}
\hline \multirow[b]{2}{*}{ Variables } & & \multicolumn{3}{|c|}{ Univariate analysis $^{\mathrm{b}}$} & \multicolumn{3}{|c|}{ Multivariate analysis ${ }^{\mathrm{c}}$} \\
\hline & & HR & $95 \% \mathrm{CI}$ & P-value & HR & $95 \% \mathrm{CI}$ & P-value \\
\hline Age & $<70$ or $\geq 70$ years & 1.92 & $0.268-13.67$ & 0.409 & & & \\
\hline Initial PSA & $<20$ or $\geq 20 \mathrm{ng} / \mathrm{ml}$ & 0.70 & $0.225-2.178$ & 0.615 & & & \\
\hline Clinical T stage & $\mathrm{T} 1-2$ or $\mathrm{T} 3$ & 3.43 & $0.352-33.370$ & 0.318 & & & \\
\hline Biopsy Gleason score & 8 or higher & 11.5 & $1.158-114.664$ & $0.004^{\mathrm{d}}$ & 9.05 & $0.427-191.9$ & 0.157 \\
\hline Neoadjuvant ADT & - or + & 0.891 & $0.122-6.525$ & 0.982 & & & \\
\hline Adjuvant ADT & - or + & 0.858 & $0.117-6.315$ & 0.707 & & & \\
\hline Adjuvant radiotherapy & - or + & 0.668 & $0.069-6.472$ & 0.417 & & & \\
\hline $\mathrm{pN}^{\mathrm{a}}$ & 0 or 1 & 4.95 & $0.448-54.604$ & 0.194 & & & \\
\hline Capsular penetration $^{\mathrm{a}}$ & - or + & 0.288 & $0.030-2.786$ & 0.278 & & & \\
\hline Seminal vesicle invasion ${ }^{a}$ & - or + & 3.92 & $0.549-27.944$ & 0.086 & & & \\
\hline Positive surgical margin ${ }^{a}$ & - or + & 1.05 & $0.146-7.477$ & 0.826 & & & \\
\hline Ki-67 staining index & $<10$ or $\geq 10 \%$ & 6.69 & $0.695-64.364$ & $0.021^{\mathrm{d}}$ & 1.26 & $0.060-26.74$ & 0.881 \\
\hline p53 &,- \pm or + & 6.45 & 0.583-71.289 & 0.079 & & & \\
\hline bcl-2 &,,- \pm+ or ++ & 19.4 & $2.012-186.521$ & $0.0001^{\mathrm{d}}$ & 13.8 & $0.938-201.9$ & 0.055 \\
\hline
\end{tabular}

C, Overall survival

\begin{tabular}{|c|c|c|c|c|c|c|c|}
\hline \multirow[b]{2}{*}{ Variables } & & \multicolumn{3}{|c|}{ Univariate analysis $^{\mathrm{b}}$} & \multicolumn{3}{|c|}{ Multivariate analysis ${ }^{\mathrm{c}}$} \\
\hline & & $\mathrm{HR}$ & $95 \% \mathrm{CI}$ & P-value & HR & $95 \% \mathrm{CI}$ & P-value \\
\hline Age & $<70$ or $\geq 70$ years & 0.763 & $0.229-2.546$ & 0.068 & & & \\
\hline Initial PSA & $<20$ or $\geq 20 \mathrm{ng} / \mathrm{ml}$ & 0.803 & $0.405-1.592$ & 0.320 & & & \\
\hline Clinical T stage & T1-2 or T3 & 1.06 & $0.312-3.596$ & 0.656 & & & \\
\hline Biopsy Gleason score & 8 or higher & 1.80 & $0.455-7.127$ & 0.187 & & & \\
\hline Neoadjuvant ADT & - or + & 1.12 & $0.347-3.965$ & 0.667 & & & \\
\hline Adjuvant ADT & - or + & 0.925 & $0.232-3.686$ & 0.566 & & & \\
\hline Adjuvant radiotherapy & - or + & 0.869 & $0.228-3.315$ & 0.154 & & & \\
\hline $\mathrm{pN}^{\mathrm{a}}$ & 0 or 1 & 2.12 & $0.247-18.17$ & 0.543 & & & \\
\hline Capsular penetration ${ }^{\mathrm{a}}$ & - or + & 0.586 & $0.174-1.973$ & 0.195 & & & \\
\hline Seminal vesicle invasion ${ }^{\mathrm{a}}$ & - or + & 0.897 & $0.193-4.164$ & 0.644 & & & \\
\hline Positive surgical margin ${ }^{\mathrm{a}}$ & - or + & 0.913 & $0.260-3.209$ & 0.801 & & & \\
\hline Ki-67 staining index & $<10$ or $\geq 10 \%$ & 1.25 & $0.364-4.314$ & 0.119 & & & \\
\hline p53 &,- \pm or + & 12.8 & $2.575-63.726$ & $0.0001^{\mathrm{d}}$ & 13.3 & $2.635-67.3$ & $0.0017^{\mathrm{d}}$ \\
\hline bcl-2 &,,- \pm+ or ++ & 2.64 & $0.678-10.25$ & $0.019^{\mathrm{d}}$ & 2.78 & $0.695-11.11$ & 0.148 \\
\hline
\end{tabular}

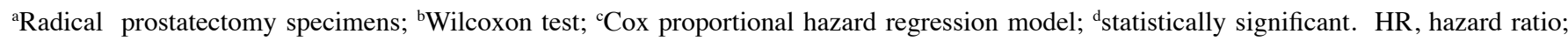
CI, confidence interval; PSA, prostate specific antigen; ADT, androgen deprivation therapy. 
biopsy specimens, however, are ideal for an array using SA technology, as the entire biopsy specimen can be represented in a reel on the array. This process is enabled by rearranging the pieces of small tissue on the plane paraffin sheet before reeling. In this context, SA examinations for breast biopsies have already been constructed (14). Up to 30, 4-mm-thick sections can be cut from an SA block holding 20 breast cancer core needle biopsy specimens. The clinical implications for evaluating diagnostic and prognostic biomarkers in needle biopsy specimens are significant. Our method allows for the maintenance of donor tissue block integrity and facilitates the collection of tissue samples, which will promote interinstitutional collaboration and, ideally, clinical applications.

There are some limitations associated with this study. The study design is retrospective and the sample size is relatively small compared to other TA studies. The cancer segments were collected only if they were longer than $3 \mathrm{~mm}$ in a needle biopsy specimen. The patient cohort was relatively heterogenous because this study included both localized and locally advanced prostate cancer patients treated with or without neoadjuvant or adjuvant androgen deprivation and with or without adjuvant radiotherapy. The Gleason grades of the prostatectomy specimens were not analyzed because $45 \%$ of the patients underwent neoadjuvant androgen deprivation, in which histological grading was not appropriate. Therefore, the results may have been different if the study design had been prospective and employed a larger and more homogeneous cohort with analyses including smaller cancer segments. The pathological findings differ between samples obtained with prostate needle biopsies and those obtained with radical prostatectomy $(24,25)$. This is one of the problems in selecting a treatment modality, which is usually chosen based on the biopsy Gleason score, clinical stage and patient's status. In this context, the present study found the p53 expression in the SA to be an independent prognostic molecular marker. This means that exploring molecular markers based on the findings of core needle biopsies not the final pathology may be possible in further studies. Another potential criticism of the SA technique concerns the limited representation of tissue morphology due to the contorted shape of the samples. However, we previously reported that the SA is more representative of the entire tumor morphology than the CTA. In that study, the results were statistically better for the SA than the CTA in identifying the organ and were identical in predicting the histologic diagnosis (14). In the present study, the SA accommodated 20 cores in one paraffin block, which is less than that observed in a CTA block. However, the increased representation of tumor heterogeneity may alleviate the need to sample multiple cores of one donor block, as is routinely performed in CTA, in which $\sim 600-800$ cores are assembled from 200 to 300 patients. With respect to multiplex cores, several reports have indicated that there is reasonable representation of tissue heterogeneity $(26,27)$. In the present study, the SA was not compared with the CTA, unlike in our previous study (14). Therefore, further studies are needed to compare these two techniques using radical prostatectomy specimens.

In conclusion, in the present study, a spiral array was applied to prostate cancer research. This is a novel approach for tissue array construction that has several advantages over the conventional construction of tissue arrays while maintaining the advantages of high-throughput array technology. The SA allows for the inclusion of surgical pathology specimens that have previously been unamenable to research owing to their limited material. The level of destruction of the original block may not be an issue for tissue arrays using small cores, such as those measuring $1.2 \mathrm{~mm}$ in diameter, obtained by prostate needle biopsies described in the present study. The SA may allow researchers to expand the investigation of entirely new tissue repositories. We believe that the SA has the potential to be a new tool for translational research on prostate cancer.

\section{Acknowledgements}

Dr J. Fukuoka received a research fund from Sakura Finetek Japan Inc.

\section{References}

1. Kononen J, Bubendorf L, Kallioniemi A, et al: Tissue microarrays for high-throughput molecular profiling of tumor specimens. Nat Med 4: 844-847, 1998.

2. Kallioniemi OP, Wagner U, Kononen J and Sauter G: Tissue microarray technology for high-throughput molecular profiling of cancer. Hum Mol Genet 10: 657-662, 2001.

3. Braunschweig T, Chung JY and Hewitt SM: Perspectives in tissue microarrays. Comb Chem High Throughput Screen 7: 575-585, 2004.

4. Tsurutani J, Fukuoka J, Tsurutani H, et al: Evaluation of two phosphorylation sites improves the prognostic significance of Akt activation in non-small-cell lung cancer tumors. J Clin Oncol 24: 306-314, 2006.

5. Fukuoka J, Dracheva T, Shih JH, et al: Desmoglein 3 as a prognostic factor in lung cancer. Hum Pathol 38: 276-283, 2007.

6. Fukuoka J, Fujii T, Shih JH, et al: Chromatin remodeling factors and BRM/BRG1 expression as prognostic indicators in non-small cell lung cancer. Clin Cancer Res 10: 4314-4324, 2004.

7. Tsuna M, Kageyama S, Fukuoka J, et al: Significance of S100A4 as a prognostic marker of lung squamous cell carcinoma. Anticancer Res 29: 2547-2554, 2009.

8. Rubin MA: Use of laser capture microdissection, cDNA microarrays, and tissue microarrays in advancing our understanding of prostate cancer. J Pathol 195: 80-86, 2001.

9. Rubin MA, Dunn R, Strawderman M and Pienta KJ: Tissue microarray sampling strategy for prostate cancer biomarker analysis. Am J Surg Pathol 26: 312-319, 2002.

10. Inoue T, Segawa T, Shiraishi T, et al: Androgen receptor, Ki67, and $\mathrm{p} 53$ expression in radical prostatectomy specimens predict treatment failure in Japanese population. Urology 66: 332-337, 2005.

11. Yasuda K, Komiya A, Watanabe A, et al: Expression of hepatocyte growth factor activator inhibitor type-1 (HAI-1) in prostate cancer. Anticancer Res 33: 575-581, 2013.

12. Komiya A, Yasuda K, Nozaki T, Fujiuchi Y, Hayashi S and Fuse H: Small cell carcinoma of the prostate after high-dose-rate brachytherapy for low-risk prostatic adenocarcinoma. Oncol Lett 5: 53-56, 2013

13. Komiya A, Yasuda K, Watanabe A, Fujiuchi Y, Tsuzuki T and Fuse $\mathrm{H}$ : The prognostic significance of loss of the androgen receptor and neuroendocrine differentiation in prostate biopsy specimens among castration-resistant prostate cancer patients. Mol Clin Oncol 1: 257-262, 2013.

14. Fukuoka J, Hofer MD, Hori T, et al: Spiral array: a new highthroughput technology covers tissue heterogeneity. Arch Pathol Lab Med 136: 1377-1384, 2012.

15. Zellweger T, Gunther S, Zlobec I, et al: Tumour growth fraction measured by immunohistochemical staining of Ki67 is an independent prognostic factor in preoperative prostate biopsies with small-volume or low-grade prostate cancer. Int J Cancer 124: 2116-2123, 2009.

16. Miyake H, Muramaki M, Kurahashi T, Takenaka A and Fujisawa M: Expression of potential molecular markers in prostate cancer: correlation with clinicopathological outcomes in patients undergoing radical prostatectomy. Urol Oncol 28: 145-151, 2010. 
17. Oxley JD, Winkler MH, Parry K, Brewster S, Abbott C and Gillatt DA: p53 and bcl-2 immunohistochemistry in preoperative biopsies as predictors of biochemical recurrence after radical prostatectomy. BJU Int 89: 27-32, 2002.

18. Ecke TH, Schlechte HH, Schiemenz K, et al: TP53 gene mutations in prostate cancer progression. Anticancer Res 30 1579-1586, 2010.

19. Nariculam J, Freeman A, Bott S, et al: Utility of tissue microarrays for profiling prognostic biomarkers in clinically localized prostate cancer: the expression of BCL-2, E-cadherin, Ki-67 and p53 as predictors of biochemical failure after radical prostatectomy with nested control for clinical and pathological risk factors. Asian J Androl 11: 109-118, 2009.

20. Cho IC, Chung HS, Cho KS, et al: $\mathrm{Bcl}-2$ as a predictive factor for biochemical recurrence after radical prostatectomy: an interim analysis. Cancer Res Treat 42: 157-162, 2010.

21. Fleischmann A, Huland H, Mirlacher M, et al: Prognostic relevance of Bcl-2 overexpression in surgically treated prostate cancer is not caused by increased copy number or translocation of the gene. Prostate 72: 991-997, 2012.

22. Abdollah F, Suardi N, Cozzarini C, et al: Selecting the optimal candidate for adjuvant radiotherapy after radical prostatectomy for prostate cancer: a long-term survival analysis. Eur Urol 63 : 998-1008, 2013.
23. Kumar S, Shelley M, Harrison C, Coles B, Wilt TJ and Mason MD: Neo-adjuvant and adjuvant hormone therapy for localised and locally advanced prostate cancer. Cochrane Database Syst Rev CD006019, 2006.

24. Chun FK, Steuber T, Erbersdobler A, et al: Development and internal validation of a nomogram predicting the probability of prostate cancer Gleason sum upgrading between biopsy and radical prostatectomy pathology. Eur Urol 49: 820-826, 2006.

25. Imamoto T, Utsumi T, Takano M, et al: Development and external validation of a nomogram predicting the probability of significant gleason sum ppgrading among Japanese patients with localized prostate cancer. Prostate Cancer 2011: 754382, 2011.

26. Camp RL, Charette LA and Rimm DL: Validation of tissue microarray technology in breast carcinoma. Lab Invest 80: 1943-1949, 2000.

27. Kyndi M, Sorensen FB, Knudsen H, et al: Tissue microarrays compared with whole sections and biochemical analyses. A subgroup analysis of DBCG 82 b\&c. Acta Oncol 47: 591-599, 2008 . 